\title{
Modelos Y Estilos De Resolución De Conflictos En El Marco De Una Cultura De La Paz Para Los Líderes De Las Unidades Habitacionales Del Municipio De Puebla, México
}

\author{
Leticia Susana Medoza Cervantes, Maestra en Desarrollo Humano \\ Susana Pérez Milicua Mendoza, Maestra en Comunicación Estratégica \\ Universidad Popular, Autónoma del Estado de Puebla (UPAEP), México
}

Doi:10.19044/esj.2018.v14n23p178 URL:http://dx.doi.org/10.19044/esj.2018.v14n23p178

\begin{abstract}
This paper focuses on the different theories on management and styles of conflict resolution in order to establish a culture of peace among the leaders of the housing units of the municipality of Puebla, Mexico. The literature was reviewed using keywords in different databases. The main findings suggest that there is a great diversity of models and styles of conflict resolution, as well as different instruments to evaluate them. However, its focus is fundamentally oriented to organizational reality, rather than to issues of a social nature. The model and style of conflict resolution that best explains the prevailing situation in housing units is the two-dimensional approach with four styles.
\end{abstract}

Keywords: Culture of peace, Conflict resolution styles, Conflict management models, Housing units

\section{Resúmen}

El propósito de esta investigación, consiste en exponer un marco de referencia sobre los principales modelos y estilos de resolución de conflictos, con la finalidad de establecer una cultura de la paz entre los líderes de las unidades habitacionales del municipio de Puebla, México. Se realizó revisión de la literatura empleando palabras claves en diferentes bases de datos. Los principales hallazgos sugieren que existen una gran diversidad de modelos y estilos de resolución de conflictos, así como distintos instrumentos para evaluarlos. Sin embargo, su enfoque está orientado fundamentalmente a la realidad organizacional, más que a temas de carácter social. El modelo y estilo de resolución de conflictos que mejor explica la situación imperante en 
unidades habitacionales es el de aproximación bidimensional con cuatro estilos.

Palabras Clave: Cultura para La Paz, Estilos de resolución de conflicto, Modelos de gestión de conflicto, Unidades habitacionales

\section{Introduction}

A nivel mundial, las políticas de vivienda en el mundo se dividen en dos conceptos claves: enabling, traducido como habilitación o facilitación, y partnership que significa asociación de distintos actores, explicado como asociación.

El primero -enabling-, hace referencia a la necesidad de suministrar un marco legislativo, institucional y financiero, en el cual los emprendimientos del sector privado de las organizaciones sociales y de los individuos, puedan desarrollar efectivamente el sector de la vivienda. El país debe contribuir a estructurar ese marco, cumpliendo un rol de facilitador de las iniciativas particulares en el ámbito del mercado.

En este sentido, el Estado debe dejar de lado su anterior función de productor de vivienda y asumir el papel de administrador en la totalidad del sector y de orientador del desempeño del ámbito privado estructurado y no estructurado. Lo anterior, supone poner más énfasis en la función global que cumple la vivienda en el desarrollo económico y social.

El segundo -partnership-, alude a la creación de nuevos escenarios de gestión que permitan aunar la acción de los gobiernos, especialmente los locales, con las iniciativas del sector empresarial y las de la sociedad civil (Izquierdo, 2007).

La situación de la vivienda, es una de las problemáticas más preocupantes de las ciudades en Latinoamérica. Actualmente, el 70\% de la población vive en zonas urbanas. Uno de los antecedentes principales que produjeron la necesidad de vivienda fue la migración del campo a la ciudad por causa de la industrialización a principios del siglo XX. Hacia la década de los setenta, la población urbana pasó de 35\% en el año 1940 a $58.7 \%$ en el año 1970 son evidentes los problemas de densidad, infraestructura y hacinamiento que enfrentaron las incipientes ciudades del país y que causaron distintas acciones políticas (Consejo Nacional de Evaluación de la Política de Desarrollo Social, 2014).

Esta situación, condujo a los profesionales de la construcción desde las primeras décadas del siglo XX, a incursionar en el diseño habitacional destinado a las clases trabajadoras del país, apareciendo las grandes unidades habitacionales de interés social, auspiciadas por el Estado. Éstas intentaban responder a ciertos parámetros universales, atendiendo a la problemática de la demanda a una población creciente y carente de recursos (Merchand, 2016). 
Esto se plasma en las políticas de vivienda que se formulan y ejecutan en los países en desarrollo. De manera simplificada, podría decirse que hay dos tendencias en curso: una hegemónica y otra, marginal. La primera tendencia, está representada por un nuevo modelo de política de vivienda que ha comenzado a imponerse en la mayor parte de los países en desarrollo y que apuesta fuerte a que los mercados funcionen.

Sus pilares básicos son la eliminación de las entidades públicas de la construcción y financiamiento, concentrándose los esfuerzos gubernamentales en lograr el mejor desempeño del sector privado. Además, el perfeccionamiento del mercado, mediante una serie de medidas e instrumentos que servirían para convertir los préstamos de vivienda en alternativas atractivas para las entidades financieras, la estabilidad macroeconómica, el fortalecimiento de la estructura legal para el financiamiento de la vivienda, la atención a las leyes que rigen para ejecutar hipotecas, la simplificación del registro de títulos de propiedad de la tierra, entre otras. También, el buen diseño de subsidios de demanda, subsidios transparentes separados de las fuentes de financiación para evitar el desgaste de las instituciones de financiamiento y tener mejor impacto en la población pobre (Cuenya, 2010).

La segunda tendencia, está representada por el conjunto de experiencias alternativas que, especialmente varios gobiernos locales, ponen en marcha en sus ciudades. Guiadas por el principio de que la ciudad debe ser accesible a todos sus habitantes, estas experiencias exigen un control institucional sobre el uso especulativo del suelo, así como una distribución y accesibilidad más equitativa de equipamientos sociales y servicios públicos (Cuenya, 2010).

Las unidades habitacionales, se distinguen por contar con dos tipos de espacios: los de uso privado (doméstico) y los de uso colectivo (áreas comunes). Lo anterior, en ocasiones ha derivado en diversos problemas, debido a que sus habitantes se olvidan de cuidar las áreas comunes y sólo se ocupan del espacio que les brinda privacidad y seguridad (Consejo Nacional de Evaluación de la Política de Desarrollo Social, 2014).

El mundo actual se mueve entre dos paradojas: la búsqueda y anhelo de una cultura de paz basada en el fomento de la democracia, el desarrollo y los derechos humanos, o la proliferación de conflictos armados internos, a los que la Organización de Naciones Unidas (ONU), debe enfrentarse pese a la reticencia de los Estados (Izquierdo, 2007).

Por lo tanto, el objetivo de este trabajo consiste en exponer un marco de referencia sobre los principales modelos y estilos de resolución de conflictos, con la finalidad de establecer una cultura de la paz entre los líderes de las unidades habitacionales del municipio de Puebla, México.

El presente artículo está estructurado en siete apartados. En el primero, se define el término de unidades habitacionales y se muestran algunas de sus 
principales características. En el segundo, se establece un marco general sobre la cultura de la paz. En el tercero, se exponen los principales elementos que convergen en el conflicto. En el cuarto, se presentan los principales modelos de resolución de conflictos. En el quinto, se exponen los principales estilos de resolución de conflictos. En el sexto, se muestra el marco de trabajo. Finalmente, en el séptimo, se presentan las principales conclusiones del estudio.

\section{Unidades habitacionales}

La unidad habitacional, es un asentamiento humano promovido por instituciones del Estado, a través del Instituto del Fondo Nacional de la Vivienda para los Trabajadores (INFONAVIT), y el Fondo de la Vivienda del Instituto de Seguridad y Servicios Sociales de los Trabajadores del Estado (FOVISSSTE), o por empresas privadas. Se caracterizan por tener una arquitectura, fachada homogénea y por estar construidos en serie ya sea dúplex, triplex, multiplex, o unifamiliar.

Los habitantes ubicados en este tipo de espacios, se caracterizan por ser trabajadores sindicalizados, de bajos ingresos y asalariados, con acceso a financiamiento de vivienda como parte de una prestación laboral, o créditos mixtos a través de una institución bancaria (Plan Integral de Atención a Unidades Habitacionales del Municipio de Puebla, 2011).

Según datos de la Comisión Nacional de Vivienda (CONAVI), el municipio de Puebla cuenta con 2,793 viviendas de tipo popular. Las cifras del INEGI apuntan que las unidades habitacionales están concentradas en un $80 \%$ en los sectores norte, noreste y sur de la ciudad, que en su mayoría fueron construidas en los años setenta (CONAVI, 2018).

Los conjuntos habitacionales se distinguen por contar con espacios de uso privado y de uso colectivo. Lo anterior, en ocasiones ha derivado en diversos problemas ya que los habitantes sólo se ocupan del espacio que les brinda seguridad y privacidad.

Entre los principales se encuentran el deterioro de las áreas comunes, el cambio en los usos para los que las áreas colectivas fueron diseñadas y su progresiva apropiación (comercio), la falta de pago de cuotas, la inseguridad y delincuencia, los conflictos entre vecinos (cultura condominal), la falta de participación y de preocupación de los ocupantes por el mantenimiento general, así como la casi inexistente relación de quienes viven en los conjuntos con su barrio o colonia (Consejo Nacional de Evaluación de la Política de Desarrollo Social, 2014).

Las desarrolladoras deben tomar parte de la responsabilidad en la administración de los conjuntos habitacionales, para que influyan en los nuevos condóminos, y de este modo se haga conciencia de las obligaciones y derechos de la propiedad, a través de la capacitación directa tanto a los 
habitantes, como a los líderes de las unidades habitacionales para conferirles cierta autonomía y participación activa en las decisiones que les competen, ofreciéndoles herramientas para la gestión de conflictos, y de esta manera hacer saber de las consecuencias que hay cuando no se logra un acuerdo en la mediación (Fernández \& López, 2014).

\section{Cultura para la paz}

Desde el inicio de los tiempos, ha prevalecido el uso de la violencia, de la fuerza y del dominio (Valencia, Darío, Gutiérrez, \& Johansson, 2012). Se cuenta con una sociedad masculina, ya que las decisiones han sido adoptadas por unos cuantos varones que se han impuesto al resto de los ciudadanos. Finalmente, la paz no ha sido más que una pausa entre dos guerras, y el poder se ha mantenido gracias a la fuerza armada (Mayor, 2011).

De acuerdo a la ONU, (1999), citado en Laca, (2010), la cultura de paz, se refiere a un conjunto de valores, actitudes, tradiciones, comportamientos y estilos de vida basados en el respeto a la vida, el fin de la violencia y la promoción y la práctica de la no violencia por medio de la educación, el diálogo y la cooperación (Méndez \& Casas, 2009).

Sin embargo, es preciso contribuir a la construcción de una nueva concepción de la paz, mediante el desarrollo de una cultura, fundada en los valores universales del respeto a la vida, a la libertad, la justicia, la solidaridad, la tolerancia, los derechos humanos y la igualdad entre hombres y mujeres (Rojas \& Arapé, 2008).

Por otra parte, no se puede entender la paz exclusivamente, como la ausencia de la guerra, sino que debe existir un acercamiento una forma mucho más global, como un fenómeno dinámico, no terminal, amplio y complejo, que está más en relación con la violencia (Dios, 2009).

Sin embargo, no existe paz sobre relaciones de dominio, de sometimiento, de injusticia o de desigualdad (Boqué, Pañellas, Alguacil, \& García, 2014). Tanto en el ámbito personal como en el grupal o internacional, no existe paz cuando las relaciones interpersonales o internacionales se basan en situaciones de inequidad (Secretaría de Educación Pública, 2011).

\section{Conflicto}

El conflicto surge de cualquier forma de interacción social y, por lo tanto, resulta difícil entender el comportamiento de las personas (Rahim, 1986 citado en Nguyen y Yang, 2012). En cualquier escenario social, las habilidades de manejo de conflictos adquieren una notable relevancia, ya que son determinantes para el correcto mantenimiento de las relaciones humanas, no sólo en la esfera personal (Gottman, 1993 citado en Pérez, 2014), sino también en la profesional (Tjosvold, 1997 citado en Montes, Serrano \& Rodríguez, 2014). 
De hecho, la gestión de conflictos es una de las funciones más importantes desempeñadas por los gerentes en las organizaciones (Euwema, Kop, \& Bakker, 2009), siendo una de las inversiones cruciales para alcanzar la viabilidad a largo plazo y lograr el éxito de cualquier organización.

A diferencia de otros ámbitos del comportamiento humano, el conflicto se expresa mediante múltiples conductas que se conocen como estilos de gestión de conflictos. Los estilos de gestión se relacionan con la orientación general y consistente hacia la otra parte y los temas en conflicto, manifestada en conductas observables que forman un patrón relativamente estable a lo largo del tiempo (Thomas, 1976). Estos estilos reflejan, en definitiva, las intenciones conductuales de los sujetos cuando hacen frente a una situación de conflicto (Womack, 2008).

El conflicto es un fenómeno natural en toda sociedad, es decir, se trata de un hecho consustancial a la vida en sociedad (Surdez, Magaña \& Sandoval, 2015). Asimismo, las disputas son una constante histórica, puesto que han comparecido en todas las épocas y sociedades a lo largo de los tiempos. Incluso, el cambio social que determina toda la dinámica de la vida de los seres humanos es una consecuencia que debe ser imputada de modo mayoritario, aun cuando no de manera absoluta al conflicto (Silva, 2008).

Las personas pertenecen a grupos que forman parte de sistemas sociales más amplios. En el ámbito de las organizaciones, éstas tienden a estar compuestas de muchos y diversos tipos de grupos y otras subunidades, cada uno con sus propias tareas, procesos de cambio y dinámica social. Como apuntan De Dreu y Van de Vliert (2007), los humanos son testigos de una creciente tendencia en las organizaciones hacia la descentralización y la organización del trabajo en grupos, que funcionan como unidades de trabajo autónomos.

Esta tendencia supone cambios relevantes para el grupo. Se requiere, una mayor coordinación entre sus miembros; se incrementa la interdependencia entre los mismos; se intensifican los procesos de influencia de unos miembros sobre otros; $y$, en definitiva, se incrementa la probabilidad del conflicto grupal (De Dreu \& Van de Vliert, 2007).

De hecho, e independientemente de la tendencia apuntada, el conflicto resulta inherente a la dinámica de los grupos. Cuando los miembros de un grupo deben tomar decisiones, resolver problemas y desarrollar otras actividades diversas, es inevitable que surjan divergencias en las preferencias sobre las decisiones, los métodos de trabajo, y los propios intereses de los miembros.

El conflicto surge cuando un individuo o un grupo se sienten negativamente afectados por otro individuo o grupo (Thomas, 1992 citado por Berruecos, 2015), o cuando percibe que las actividades desplegadas para la consecución de sus objetivos, son incompatibles con las actividades de otras 
personas y grupos (Deutsch, 1980 citado por Berruecos, 2015). El conflicto conlleva, por lo tanto, sentimientos de frustración, y activa la tensión y el malestar de las partes implicadas.

Sin embargo, no siempre resulta disfuncional para la dinámica del grupo. El conflicto interpersonal e intergrupal, puede reforzar más que obstaculizar el logro individual, la calidad de las decisiones del grupo y la efectividad organizacional (Janssen, Van de Vliert y Veenstra, 1996 citado por Yeung, Fung, y Chan, 2015). En organizaciones excesivamente armoniosas, o represivas en cuanto a la manifestación de las desavenencias y las protestas, la activación e intensificación pueden resultar más recomendables, que la prevención o la mitigación de las situaciones de conflicto (Van de Vliert y de Dreu, 1994 citado por Villamediana, Donado y Zerpa, 2015).

Se puede distinguir entre conflictos centrados en las tareas versus conflictos centrados en las personas. Esta perspectiva es central para comprender cuándo la estimulación del conflicto puede resultar productiva. Los conflictos centrados en las tareas, tienden a mejorar los resultados conjuntos del grupo, mientras que los conflictos centrados en las personas, juegan un rol disfuncional en la consecución de dichos resultados (Amason, 1996; Amason y Schweiger, 1994 citado por Munduate y Dorado, 2016).

Parece ser que el tipo de conflicto y el modo de abordarlo, están estrechamente relacionados. Por lo tanto, no sólo es recomendable activar e intensificar el conflicto sobre las tareas, sino prevenir y mitigar el conflicto sobre los aspectos personales. El consejo de separar a las personas de los problemas, si bien resulta de gran utilidad (Fisher y Ury, 1981 citado por Munduate y Dorado, 2015), no es infalible ya que como argumentan Van de Vliert y de Dreu, 1994 citado por Yeung, Fung, y Chan, 2015), no es fácil encontrar una total desconexión entre tareas y emociones, por lo que raramente se dan conflictos puramente laborales y conflictos puramente personales.

Además, la intensificación del conflicto puede tornar o implicar a los aspectos personales y afectivos en los aspectos de tarea, por lo que a pesar de que resulta recomendable centrarse sólo en la activación de las diferencias en cuanto a los problemas en cuestión, deben estar presentes otros condicionantes para que dicha activación resulte exitosa, como el grado de conflictividad y las tendencias en los estilos de gestión del conflicto por parte de los miembros del grupo (Pérez, 2014).

\section{Modelos de estilos de resolución de conflictos}

La resolución de conflicto se significa a la terminación del conflicto a través de métodos analíticos y que se dirigen a la raíz del problema. Resolución de conflicto, como opuesto a la gestión o ajuste, se orienta a un resultado que, de acuerdo con las partes implicadas, es una solución permanente del problema. 
Puesto que busca acceder a las fuentes de los problemas, la resolución de conflicto pretende no meramente resolver el conflicto social inmediato, la disputa inmediata ya sea familiar o étnica, sino a la vez, arrojar luz sobre la naturaleza genérica del problema y, de este modo, contribuir a la eliminación de sus fuentes y a la prevención de otros procesos (Burton, 2016).

El Modelo de Intereses Doble -Dual Concern Model- (Blake y Mouton, 1964; Pruitt, 1983; Pruitt y Rubin, 1986 citado en Montes, Serrano y Rodríguez, 2015), es el enfoque predominante utilizado para conceptualizar la preferencia o elección de los estilos de gestión de conflictos. Esta aproximación establece que las personas escogen diferentes modos, estrategias o estilos para manejar los conflictos basándose en dos motivaciones o intereses primarios -el interés propio y el interés por los demás-, que son independientes entre sí y varían en cuanto a su intensidad en un rango de débil a fuerte.

El interés propio se refiere al grado en que las partes en conflicto se centran en sus propias necesidades, intereses, valores y creencias. El interés por los demás, por su parte, se refiere a la medida en que las partes en conflicto se centran en las necesidades, intereses, valores y creencias de sus oponentes.

La Red Gerencial mejor conocida como Managerial Grid (Blake y Mouton, 1964, citado en Altmäe y Türk, 2011), representa el primer esquema conceptual que clasificó los estilos de gestión de conflictos y fue el modelo precursor del Modelo de Intereses Dobles.

Se identifican cinco modos de manejo de conflictos dependiendo del grado en que los sujetos tuviesen interés por la producción e interés por las personas. Desde su planteamiento, este modelo ha sufrido diferentes reformulaciones basadas en distintos puntos de vista.

Por un lado, se tiene el interés por la producción, el interés por los objetivos personales, el interés por los resultados propios, la asertividad y el interés propio. Por el otro lado, se toman en cuenta factores externos como el interés por las relaciones, el interés por las personas, el interés por los resultados de los demás, la cooperación así como el interés por los demás.

Todas ellas asumen de modo análogo que los sujetos escogen diferentes estrategias, modos o estilos para manejar un conflicto basándose en dos motivaciones o intereses primarios (Van de Vliert, 2007). Es ahí donde reside la principal diferencia entre todas las formulaciones, esto es cómo se conceptualizan los intereses subyacentes.

Así, el interés por la producción y el interés por las personas se han interpretado respectivamente como interés por los objetivos personales e interés por las relaciones como explica Hall (1964), y la asertividad y cooperación de Thomas (1976), o interés por los propios resultados e interés por los resultados de los demás (Pruitt, 1983; Pruitt y Rubin, 1986; citado en 
Montes, Serrano y Rodríguez, 2015). La Tabla 1 muestra una síntesis de lo planteado.

Tabla 1. Modelo de intereses dobles.

\begin{tabular}{|c|c|c|}
\hline Primer cuadrante & Segundo cuadrante & Tercer cuadrante \\
\hline $\begin{array}{l}\text { Interés por la producción } \\
\text { (Blake \& Mouton, 1964). } \\
\text { Interés por os objetivos } \\
\text { personales (Hall, 1969). } \\
\text { Interés por los resultados } \\
\text { propios (Pruitt, 1983). } \\
\text { Asertividad (Thomas, } \\
\text { 1976) } \\
\text { Interés propio (Rahim \& }\end{array}$ & $\begin{array}{l}\text { Dominación (Blake \& } \\
\text { Mouton, 1964). } \\
\text { Ganar-Perder (Hall, 1969). } \\
\text { Rivalizar (Pruitt, 1983). } \\
\text { Competición (Thomas, } \\
\text { 1976). } \\
\begin{array}{l}\text { Dominación (Rahim \& } \\
\text { Bonoma, 1979). }\end{array}\end{array}$ & $\begin{array}{l}\text { Solución de problemas } \\
\text { (Blake \& Mouton, 1964). } \\
\text { Sinérgico (Hall, 1969). } \\
\text { Solución de problemas } \\
\text { (Pruitt, 1983). } \\
\text { Colaboración (Thomas, } \\
\text { 1976). } \\
\text { Integración (Rahim \& } \\
\text { Bonoma, 1979). }\end{array}$ \\
\hline & $\begin{array}{l}\text { Compromiso (Blake \& } \\
\text { Mouton, 1964; Thomas, } \\
\text { 1976; Rahim \& Bonoma, } \\
\text { 1979). }\end{array}$ & \\
\hline $\begin{array}{l}\text { Retirada (Blake \& Mouton, } \\
\text { 1964). } \\
\text { Perder - Abandonar (Hall, } \\
\text { 1969). } \\
\text { Inanición (Pruitt, 1983). } \\
\text { Evitación (Thomas, 1976; } \\
\text { Rahim y Bonoma, 1979). }\end{array}$ & $\begin{array}{l}\text { Interés por las personas } \\
\text { (Blake \& Mouton, 1964). } \\
\text { Interés por las relaciones } \\
\text { (Hall, 1969). } \\
\text { Interés por los resultados de } \\
\text { los demás (Pruitt, 1983). } \\
\text { Interés por los demás (Rahim } \\
\& \text { Bonoma, 1979). } \\
\text { Cooperación (Thomas, } \\
\text { 1976). }\end{array}$ & $\begin{array}{l}\text { Suavizante (Blake \& } \\
\text { Mouton, 1964). } \\
\text { Ceder-Perder (Hall, 1969). } \\
\text { Complacer (Pruitt, 1983). } \\
\text { Acomodación (Thomas, } \\
\text { 1976). } \\
\text { Servilismo (Rahim \& } \\
\text { Bonoma, 1979). }\end{array}$ \\
\hline
\end{tabular}

Fuente: elaboración propia con base con base en Montes, Serrano y Rodríguez (2015).

Por su parte, Rahim y Bonoma (1979), citado en Montes, Serrano y Rodríguez (2012) reinterpretaron el interés por la producción y por las personas como interés propio e interés por los demás. Estas dimensiones retratan la orientación motivacional de los sujetos durante el conflicto (Rahim \& Magner, 1995) y, combinadas, producen cinco estilos diferentes como se expone en la Tabla 2.

Tabla 2. Orientación motivacional de los sujetos durante en conflicto de Rahim y Magner.

\begin{tabular}{cc}
\hline Orientación & Definición \\
\hline Integración & Alto interés propio y alto interés por los demás. \\
\hline Dominación & Alto interés propio y bajo interés por los demás. \\
\hline Servilismo & Bajo interés propio y alto interés por los demás. \\
\hline Evitación & Bajo interés propio y bajo interés por los demás. \\
\hline Compromiso & Moderado interés propio y moderado interés por los demás. \\
\hline
\end{tabular}

Fuente: elaboración propia con base con base en Montes, Serrano y Rodríguez (2015).

Según los autores el Dual Concern Model, ha hallado un sólido respaldo en la investigación empírica $\mathrm{y}$, a partir de la distinción teórica entre los motivos básicos que regulan el comportamiento en situaciones de 
conflicto, se han diseñado diferentes escalas por autores como: Hall, Thomas y Kilmann, Putnam y Wilson, Rahim, de De Dreu, Evers, Beersma, Kluwer y Nauta. Estos cuestionarios se ajustan a los presupuestos teóricos del Dual Concern Model y asumen que el estilo de conflicto preferido es aquel que obtiene una puntuación mayor (Altmäe \& Türk, 2011).

A pesar de ello, la relación entre las dimensiones y la elección de las estrategias desplegadas en un conflicto no han sido adecuadamente validadas. Esto es debido a que los estudios previos presentan fundamentalmente tres limitaciones. La mayoría de los investigadores no han examinado el interés propio y el interés por el oponente como base para la elección de una estrategia de conflicto. Además, algunos estudios que se centran en el interés propio y en el interés por el oponente, no miden realmente estos constructos. Los estudios que sí han medido estos intereses no han demostrado adecuadamente qué niveles e interacciones predicen la elección de una estrategia de conflictos concreta (Sorenson et al., 2009).

Durante las cinco décadas pasadas, unas variedades de teorías han aclarado la dirección del conflicto a través de diferentes estilos y sus dimensiones subyacentes, y un sinnúmero de instrumentos de medición han sido desarrollados y validados para evaluar los estilos de manejo de conflictos de los individuos (De Dreu, et al., 2001).

Esta situación ha propiciado el desarrollo de algunos instrumentos, descritos y analizados con extensión por Van de Vliert y Kabanoff (1990), y King y Miles (1990), citados en Vizcaino y Olvera (2016), que tratan de evaluar el grado en que se emplean los diversos estilos de gestión del conflicto en la realidad organizacional. La Tabla 3 expone los principales instrumentos

Tabla 3. Instrumentos de gestión de conflictos.

Instrumentos

\begin{tabular}{cc}
\hline Instrumentos & Autor (es) \\
\hline Thomas-Kilmann Conflict Mode Instrument (TKI) & Thomas y Kilmann (1974). \\
\hline $\begin{array}{c}\text { Organizational Conflict Communication Inventory } \\
\text { (OCCI). }\end{array}$ & Putman y Wilson (1982). \\
\hline Rahim Organizational Conflict Inventory (ROCI II). & Rahim (1983). \\
\hline
\end{tabular}

Fuente: elaboración propia con base en Vizcaino y Olvera (2016).

\section{Estilos de resolución de conflictos}

Los estilos de gestión del conflicto se definen como "aquellos comportamientos orientados hacia la intensificación, reducción y resolución de la tensión propiciada por el mismo" (De Dreu, Harinck y van Vianen, 1999, citado en Munduate, Ganaza y Alcaide, 2013).

Por dirección efectiva del conflicto, no se refiere sólo a la cantidad del mismo, sino también a las distintas modalidades de conducta conflictiva que pueden desplegar los participantes en una situación o episodio de conflicto.

Se han establecido, diversas tipologías relativas a los estilos de dirección del conflicto interpersonal. Según Rahim (1990), citado en 
Munduate, Ganaza y Alcaide (2013), la distinción y clasificación entre estilos se ha realizado a través de las aproximaciones que se presentan en la Tabla 4.

Tabla 4. Estilos de resolución de conflictos según Rahim.

\begin{tabular}{ll}
\hline \multicolumn{1}{c}{ Estilo } & \multicolumn{1}{c}{ Definición } \\
\hline $\begin{array}{l}\text { La aproximación de } \\
\text { dimensión única. }\end{array}$ & $\begin{array}{l}\text { Se distingue entre dos modos o estilos de conducta conflictiva: } \\
\text { la cooperación y la competición (Tjosvold, 1989). }\end{array}$ \\
\hline $\begin{array}{l}\text { La aproximación de los } \\
\text { tres estilos. }\end{array}$ & $\begin{array}{l}\text { Distingue tres modos de manejar el conflicto: la no } \\
\text { confrontación, la orientación hacia la solución y el control. }\end{array}$ \\
\hline
\end{tabular}

Tabla 4. Continuación Estilos de resolución de conflictos según Rahim.

\begin{tabular}{|c|c|}
\hline $\begin{array}{l}\text { La aproximación } \\
\text { bidimensional con } \\
\text { cuatro estilos. }\end{array}$ & $\begin{array}{l}\text { Considera que la conducta conflictiva no puede verse como una } \\
\text { conducta de una sola dimensión. Hay que tomar en } \\
\text { consideración dos dimensiones distintas, el interés por los } \\
\text { propios resultados y el interés por los resultados del otro. Por lo } \\
\text { tanto, cualquier estilo desarrollado por los sujetos será una } \\
\text { combinación de estas dos dimensiones que apuntan de bajo a } \\
\text { alto. Los estilos presentados son cuatro: solución de problemas } \\
\text {-alto interés por los resultados propios y alto interés por los } \\
\text { resultados del otro-, rivalizar -alto interés por los resultados } \\
\text { propios y bajo interés por los resultados del otro- complacer - } \\
\text { bajo interés por los resultados propios y alto interés por los } \\
\text { resultados del otro- e inacción -bajo interés por los resultados } \\
\text { propios y bajo interés por los resultados del otro- (Pruitt, 1983). }\end{array}$ \\
\hline $\begin{array}{l}\text { La aproximación de dos } \\
\text { dimensiones. }\end{array}$ & $\begin{array}{l}\text { Considera que la conducta conflictiva de una persona puede ser } \\
\text { vista como una combinación de la inclinación presentada por } \\
\text { dicha persona ante dos dimensiones diferentes. El origen de esta } \\
\text { aproximación se encuentra en la catalogación realizada por } \\
\text { Blake y Mouton (1964). Para estos autores las puntuaciones que } \\
\text { se pueden alcanzar en ambas dimensiones varían entre un } \\
\text { mínimo de } 1 \text { y un máximo de } 9 \text {, de modo que en teoría podrían } \\
\text { existir } 81 \text { estilos diferentes. Sin embargo, terminan reduciendo } \\
\text { su aportación a cinco estilos: dominar o forzar, retirada, } \\
\text { suavizante, compromiso y solución de problemas. }\end{array}$ \\
\hline
\end{tabular}

Fuente: elaboración propia con be en Munduate, Ganaza y Alcaide (2013).

La conceptualización realizada por Thomas (1976), citado en Vizcaino y Olvera (2016), es una reinterpretación de la aproximación efectuada por Blake y Mouton (1964), citado en Vizcaino y Olvera (2016). Para Thomas (1976), las dos dimensiones básicas que determinan la distinción entre los distintos modos de manejo del conflicto son el grado de asertividad mostrado por el individuo, es decir, la medida en que intenta satisfacer sus propios intereses y el grado de cooperatividad desplegado por el sujeto o el intento de satisfacer los intereses de la otra parte. En la conceptualización de Thomas, ambas dimensiones tienen dos puntuaciones extremas: asertivo vs. no asertivo y cooperador vs. no cooperador (Tabla 5). 
Tabla 5. Estilos de conducta conflictiva de Blake y Mouton.

\begin{tabular}{cc}
\hline Estilo & Definición \\
\hline Colaboración & Asertivo y cooperador. \\
\hline Competición & Asertivo y no cooperador. \\
\hline Evitación & No asertivo y no cooperador. \\
\hline Acomodación & No asertivo y cooperador. \\
\hline Compromiso & Medianamente asertivo y medianamente cooperador. \\
\hline
\end{tabular}

Fuente: elaboración propia con base en Munduate, Ganaza y Alcaide (2013).

Existe una tercera reinterpretación de los estilos de manejo del conflicto, similar a las realizadas por Blake y Mouton (1964), citado en Vizcaino y Olvera (2016), y Thomas (1976), citado en Vizcaino y Olvera (2016). Esta nueva clasificación es la realizada por Rahim y Bonoma (1986). La conceptualización diferencia los estilos de dirección del conflicto con base en dos dimensiones, el interés propio y el interés de los otros.

La primera dimensión explica la medida -alta o baja- en que un determinado sujeto trata de satisfacer su propio interés. En tanto que la segunda dimensión, explica la medida -alta o baja- en que un individuo quiere o desea satisfacer el interés de los demás.

Podría decirse que estas dos dimensiones ponen de manifiesto las orientaciones motivacionales de una determinada persona durante la situación de conflicto. Desde esta perspectiva, los cinco estilos posibles son los que se muestran en la Tabla 6.

Tabla 6. Estilos de gestión de Rahim y Bonoma.

\begin{tabular}{cl}
\hline Estilo & \multicolumn{1}{c}{ Definición } \\
\hline \multirow{3}{*}{ Integración } & $\begin{array}{l}\text { Alto interés por uno mismo y los demás. Es un estilo que implica la } \\
\text { colaboración entre los actores, por lo que puede llevar a la apertura, el } \\
\text { intercambio de información y el examen de las diferencias existentes para } \\
\text { tratar de llegar a una solución que sea aceptable para ambas partes. Este } \\
\text { estilo supone la comunicación directa entre las partes, lo que posibilita la } \\
\text { resolución de problemas, conduciendo a soluciones creativas e } \\
\text { imaginativas para los mismos. }\end{array}$ \\
\hline \multirow{5}{*}{ Servilismo } \\
$\begin{array}{l}\text { Bajo interés por uno y alto interés por los demás. Cuando una persona } \\
\text { adopta este estilo, opta por no tomar en cuenta las diferencias existentes } \\
\text { con la otra parte y se centra en enfatizar y destacar los aspectos comunes } \\
\text { existentes entre ambos para procurar satisfacer el interés del otro actor. } \\
\text { Supone en cierto grado un sacrificio para el protagonista que adopta este } \\
\text { estilo. }\end{array}$ \\
$\begin{array}{l}\text { Alto interés por uno y bajo interés por los demás. Es un estilo que ha sido } \\
\text { identificado con una orientación de ganador/perdedor. Se trata de un tipo } \\
\text { de persona que procurará por todos los medios lograr su objetivo y que, } \\
\text { como consecuencia de ello, ignorará bastante a menudo las necesidades } \\
\text { yominación expectativas de su oponente. }\end{array}$ \\
\hline
\end{tabular}


Tabla 6. Estilos de gestión de Rahim y Bonoma.

Bajo interés por los resultados propios y por los de los demás. Claramente asociada con la retirada y la esquivación del problema, a veces adopta la

Evitación postura de posponer el problema hasta un momento más propicio. En otras ocasiones es la simple retirada de una situación que resulta amenazadora.

Estilo intermedio en interés propio y de los demás. Las partes ceden algo

Tendencia al en su posición para poder tomar una decisión mutuamente aceptable para

compromiso ambas. A veces significa intercambio de concesiones, otras la búsqueda de una posición intermedia.

Fuente: elaboración propia con base con base en Montes, Serrano y Rodríguez (2015).

\section{Marco de trabajo}

Se trata de una investigación de revisión literaria, cuyo propósito consiste en exponer un marco de referencia sobre los principales modelos y estilos de resolución de conflictos, con la finalidad de establecer una cultura de la paz entre los líderes de las unidades habitacionales del municipio de Puebla, México.

El criterio empleado para las búsquedas en bases de datos fueron las palabras clave. Las principales estrategias de búsqueda utilizadas fueron: gestión de conflictos, cultura de la paz, estilos y modelos de resolución de conflictos. Con la finalidad de establecer un marco de referencia más amplio, el estudio recogió una gran diversidad de estudios desarrollados en diferentes contextos y enfoques.

Las principales tareas desarrolladas para efectos del estudio, consisten en un primer análisis de los objetivos y hallazgos de los documentos recuperados por medio de palabras clave. Posteriormente, se revisaron a profundidad los documentos y para su discriminación, se utilizaron criterios tales como año de publicación, conceptos y referencias. Finalmente, se realizó la construcción del documento.

\section{Conclusion}

La cultura para la paz, se fomenta a través de la resolución y la gestión de conflictos, por lo que, mediante la recolección de distintos modelos y estilos expuestos en el trabajo, se puede asegurar que estos temas se encuentran fuertemente relacionados, lo cual aplicado a las necesidades de los líderes de las unidades habitacionales de Puebla México, se puede generar una convivencia armónica entre los integrantes de dichas unidades.

Es una realidad que la convivencia social no es un tema sencillo ya que cada individuo posee un cúmulo de deseos y necesidades diferentes. En ocasiones, lo que favorece a uno, puede perjudicar a otro. Por lo tanto, la comprensión de los diferentes modelos y estilos de resolución de conflictos, puede favorecer el establecimiento de una cultura de la paz. 
Los hallazgos sugieren que existen una gran diversidad de modelos y estilos de resolución de conflictos, así como distintos instrumentos para evaluarlos. Sin embargo, su enfoque está orientado fundamentalmente a la realidad organizacional, más que a temas de carácter social, como lo es el caso de las unidades habitacionales. También, los hallazgos indican que el tipo de conflicto y el modo de abordarlo, se encuentran ampliamente vinculados.

Por lo tanto, el modelo y estilo de resolución de conflictos que mejor explica la situación imperante en las unidades habitacionales del municipio de Puebla, México debido a su cultura y el contexto en el que se encuentran, es el propuesto por Rahim (1986). En específico el estilo sobre la aproximación bidimensional con cuatro estilos, ya que considera el interés por los propios resultados y el interés por los resultados del otro, por lo que cualquier estilo desarrollado por los sujetos, será una combinación de estas dos dimensiones.

Sin embargo, se deben desarrollar instrumentos de carácter social que respondan a interacciones personales en otro tipo de circunstancias y espacios, ya que los propuestos por Thomas y Kilmann, Putman y Wilson, así como Rahim, se encuentran orientados a temas organizacionales.

\section{References:}

1. Altmäe, S. Y. \& Türk, K. (2011). Thomas-Kilmann's Conflict Management Modes and their relationship to Fiedler's Leadership Styles (basing on Estonian organizations). Baltic Journal of Management, Vol. 8 No. 1, 2013, pp. 45-65

2. Amason, A. C. (1996). Distinguishing the effects of functional and dysfunctional conflict on strategic decision making: Resolving a paradox for top management teams». Academy of Management Journal, 39 (1), pp. 123-148.

3. Amason, A. C. \& Schweiger, D. M. (1994). Resolving the paradox of conflict, strategic decision making, and organizational performance». The International Journal of Conflict Management, 5, 239-253.

4. Acevedo, A. Y. \& Rojas, Z. (2016). Generalidades del conflicto, los preceso de paz y el posconflicto. Revista de la facultad de derecho y ciencias políticas- UPB, Vol.46. No.124.

5. Blake, R.R. \& Mouton, J.S. (1964). The Managerial Grid. Houston: Gulf.

6. Berruecos, L. (2015). H. Max Gluckman, las teorías antropológicas sobre el conflicto y la escuela de Manchester. El Cotidiano 153. Universidad Autónoma del Estado de México. 97-113.

7. Boqué, M., Pañellas, M., Alguacil, M., \& García, L. (2014). La cultura de paz en la educación para la ciudadanía y los derechos humanos en los libros de texto de educación primaria. Perfiles Educativos, vol. XXXVI, núm. 146, 2014, IISUE-UNAM. 
8. Burtón, J. (2016). La resolución de conflictos como sistema político. [En línea], disponible en: http://www.comunicacionfamiliar.com/biblioteca1/ResoluciondeConf licto_Burton.pdf. Consultado: 7 de noviembre 2017.

9. Consejo Nacional de Evaluación de la Política de Desarrollo Social / CONEVAL (2014). Evaluación y diagnóstico [En línea], disponible en:

http://www.coneval.org.mx/Informes/Evaluacion/Diagnostico/Diagn ostico_2014/Diagnostico_2014_SEDATU_S253.pdf Consultado: 7 de noviembre 2017, p.1-43.

10. Comisión Nacional de Vivienda / CONAVI (2017). Inventario de Vivienda Vigente por Valor de la Vivienda. [En línea], disponible en: http://www.conavi.gob.mx:8080/Reports/Inv_Viv_Vig/Inv_x_TipVi v.aspx. Consultado: 7 de noviembre 2017.

11. Cuenya, B. (2010). Cambios, logros y conflictos en la política de vivienda en Argentina hacia fines del siglo XX. Boletín CF+S 29/30. Notas para entender el Mercado Inmobiliario.

12. De Dreu, C.K.W., Evers, A., Beersma, B., Kluwer, E.S., \& Nauta, A. (2001). A theorybased measure of conflict management strategies in the workplace. Journal of Organizational Behavior, 22, 645-668.

13. De Dreu, C. K. W., \& van Vianen, A. E. M. (2001). Managing relationship conflict. Journal of Organizational Behavior, 22(3), 309328.

14. De Dreu, C. \& Van De Vliert, E. (EDS.) (2007). Using Conflict inOrganizations. London: Sage.

15. Deutsch, M. (1949). A theory of cooperation and competition. Human Relations, 2, pp. 129- 151.

16. Deutsch, M. (1980). Fifty years of conflict En L. Festinger (ed.), Retrospection and Social Psychology. New York: Oxford University Press.

17. Dios, M. (2009). Redes de movimientos pacifistas. La educación y la cultura de la paz: el currículum de la noviolencia. Tiempo de Paz, \#192. España. PP. 21-31.

18. Euwema, M.C., Kop, N., \& Bakker, A.B. (2009). Burnout and dominance in profesional interactions. Work \& Stress, 18, 1-16.

19. Fernández, A. \& López, M.C. (2014). Educar para la paz. Necesidad de un cambio epistemológico Convergencia. Revista de Ciencias Sociales, vol. 21, núm. 64, enero-abril, 2014, pp. 117-142 Universidad Autónoma del Estado de México,Toluca, México.

20. Fisher, R. \& Ury, W. (1981). Obtenga el Sí. El arte de negociar sin ceder. México: CECSA. 
21. Gottman, J.M. (1993). The role of conflict engagement, escalation, and avoidance in marital interaction: A longitudinal view of five types of couples. Journal of Consulting and Clinical Psychology, 61(1), 6-15.

22. Instituto Nacional de Estadística y Geografía (2017). Zonas Urbanas, [En línea], disponible en: http://www.inegi.org.mx. Consultado: 7 de noviembre 2017.

23. Izquierdo, L. (2007). Perspectiva de los conjuntos habitacionales en México. Revista de Psicología, Vol.3. 47-56.

24. Janssen, O., Van De Vliert, E., \& Veenstra, C. (1996). Productive conflict in management teams: How and when positive interdependencecount, comunicación presentada al Ninth Annual Conference of theInternational Association for Conflict Management, Ithaca, New York, junio 2-5, 1996.

25. Laca, F. (2010). Cultura de Paz y Psicología del Conflicto, Época II. Vol. XII. Núm. 24, Colima, diciembre 2010, pp. 55-70

26. Méndez, N. \& Casas, A. (2009). Educación para la paz, cultura política y cambio social: un análisis empírico del programa Aulas en Paz desde el institucionalismo cognitivo. Desafíos, \#21, Semestre II. Colombia. PP. 97-134.

27. Merchand, M. (2016). Estado, vivienda de interés social e inmobiliarias en México. Cuadernos de Vivienda y Urbanismo, 10(19), xx-xx, Universidad Javeriana.

28. Montes, C., Rodríguez, D., \& Serrano, G. (2012). Affective choice of conflicto management styles, International Journal of Conflict Management, Vol. 23 Issue: 1, pp.6-18.

29. Montes, C., Serrano, G., \& Rodríguez, D. (2014). Estrategias de manejo de conflicto en clave emocional Anal. Psicol. vol.30 no.1 Murcia Enero 2014.

30. Montes, C., Serrano, G., \& Rodríguez, D. (2015). Impacto de las motivaciones subyacentes en la elección de las estrategias de conflicto. Boletín de Psicología, No. 100, noviembre 2010, 55-69

31. Munduate, L. Y. \& Dorado, M.A. (2015). El conflicto en los grupos de trabajo. Aprendizaje. Revista de Psicología Social, 15 (1) 30-39.

32. Munduate, L., Ganaza, J. Y., \& Alcaide, M. (2013). Estilos de gestión del conflicto interpersonal en las organizaciones. Aprendizaje. Revista de Psicología Social, 8 (1), 47-56.

33. Nguyen, H. Y. \& Yang, J. (2012). Chinese employees' interpersonal conflict management strategies, International Journal of Conflict Management, Vol. 23 Issue: 4, pp.382-412.

34. Organización de las Naciones Unidas / ONU. (1999). Declaración y programa de acción sobre una cultura de paz. Tiempo de paz, \#99. España. PP. 111-116. 
35. Pérez, M. (2014). Estilos de manejo de conflicto como estrategia de gerencia en el personal ejecutivo en empresas siderúrgicas a nivel nacional. Forum Humanes, Vol 3, No. 1.

36. Plan Integral de Atención a Unidades Habitacionales del Municipio de Puebla / PIANUH. (2011). Plan Integral de Atención a Unidades Habitacionales del Municipio de Puebla. Puebla Gobierno Municipal.

37. Pruitt, D.G. (1983). Strategic choice in negotiation. American Behavioral Scientist, 27(2), 167-194.

38. Pruitt, D.G. \& Rubin, J.Z. (1986). Social Conflict: Escalation, Stalemate, and Settlement. New York: Random House.

39. Rahim, M.A. (1986). Managing Conflicts in Organizations. New York: Praeger.

40. Rojas, L.R. \& Arapé, E. (2008). Comunicación humana y cultura de paz: una aproximación teórica. LÓGOI Revista de Filosofía, \#14. Universidad Católica Andrés Bello: Venezuela. PP. 109-125.

41. Secretaría de Desarrollo Urbano y Vivienda (2014). Programa de Reordenamiento y Rescate de Unidades Habitacionales. México.

42. Secretaría de Educación Pública, y Subsecretaría de Educación Básica I SEP. (2008). Curso de mediación de conflictos y prevención de la violencia y las adicciones en escuelas de educación básica. FLASCO: México.

43. Silva, G. (2008). La teoría del conflicto, un marco teórico necesario. Prolegómenos - Derechos y Valores Bogotá, D.C., Colombia Volumen XI - No 22 - julio - diciembre 2008.

44. Sorenson, R.L., Morse, E.A., \& Savage, G.T. (2009). A test of the motivations underlying choice of conflict strategies in the DualConcern Model. International Journalof Conflict Management, 10(1), 25-44.

45. Surdez, E., Magaña, D., \& Sandoval, M. (2015). Conflicto de rol en profesores universitarios integrantes de cuerpos académicos. Perfiles Educativos, vol. XXXVII, núm. 147, 2015, IISUE-UNAM.

46. Thomas, K.W. \& Kilmann, R.H. (1974). Thomas-Kilmann Conflict Mode Instrument. Tuxedo, NY: Xicom, Inc.

47. Tjosvold, D. (1997). Conflict within interdependence: its value for productivity and individuality. En De Dreu, C.K.W. y Van de Vliert, E. (Eds.), Using conflicts in organizations (pp. 23-37). Thousand Oaks: Sage.

48. Valencia, G., Dario, G., Gutiérrez, A., \& Johansson, S. (2012). Negociar la paz: una síntesis de los estudios sobre la resolución negociada de conflictos armados internos. Estudios Políticos, 40, Instituto de Estudios Políticos, Universidad de Antioquia, (p. 149-174). 
49. Van de Vliert, E. (2007). Complex interpersonal behavior: Theoretical frontiers. East.

50. Villamediana, J., Donado, A., \& Zerpa, C.E. (2015). Estilos de manejo de conflictos, inteligencia emocional y desarrollo moral. Revista Dimensión Empresarial, vol. 13, núm. 1, p. 73-94

51. Vizcaino, M. \& Olvera, R. (2016). Manejo de Conflictos en las Organizaciones el caso CANACO SERVYTUR. Revista de la Universidad Latina de México, febrero 2016, 3-30.Sussex, UK: Psychology Press.

52. Womack, D. F. (2008). Assessing the Thomas-Kilmann Conflict Model Survey. Management Communication Quarterly, 1(3), 321-349.

53. Yeung, D., Fung, H., \& Chan, D. (2015). Managing conflict at work: comparison between younger and older managerial employees, International Journal of Conflict Management, Vol. 26 Issue: 3, pp.342-364. 